\title{
Covid-19 und die lateinamerikanischen Arbeitsmärkte
}

Jürgen Weller

Die Covid-19 Pandemie erzeugte massive Verwerfungen auf den lateinamerikanischen Arbeitsmärkten. Aufgrund der Besonderheiten dieser Krise - vor allem mit Blick auf die Einschränkungen in den Bereichen Wirtschaft, Bildung und Mobilität der Erwerbsbevölkerung - konnten die bei Wirtschaftskrisen üblichen Anpassungsmechanismen dieser Arbeitsmärkte nicht funktionieren. Im Ergebnis verzeichnete Lateinamerika (einschließlich der Karibik) neben einer Schrumpfung des regionalen Bruttosozialprodukts um 6,8 Prozent im Jahr 2020 (CEPAL 2021) einen massiven Rückgang der geleisteten Arbeitsstunden von geschätzten 16,2 Prozent. Damit war Lateinamerika weltweit die Region mit dem größten Rückgang des Arbeitsvolumens (ILO 2021). ${ }^{1}$

In diesem Beitrag werden zunächst die Auswirkung der von der Pandemie ausgelösten Krise auf den lateinamerikanischen Arbeitsmärkten dargestellt, wobei hervorgehoben wird, wie verschiedene Gruppen von Erwerbstätigen unterschiedlich getroffen wurden und wie dies die Ungleichheit auf diesen Märkten weiter vertiefte. Im darauf folgenden Abschnitt wird die graduelle Erholung der Arbeitsmärkte ab dem zweiten Halbjahr 2020 dokumentiert, mit der bis Anfang 2021 jedoch noch nicht das Beschäftigungsniveau vor der Krise erreicht wurde. Im dritten Abschnitt wird auf einige längerfristige, vor allem mit digitalen Technologien verknüpfte Tendenzen auf dem Arbeitsmarkt verwiesen, die durch die Pandemie beschleunigt wurden. Schließlich werden die wichtigsten Resultate dieses Artikels zusammengefasst.

\section{Massive Verwerfungen auf dem Arbeitsmarkt}

Die Entwicklung der lateinamerikanischen Arbeitsmärkte vor dem Ausbruch der Pandemie durch Covid-19 war von niedrigem Wirtschaftswachstum und einer geringen Schaffung von Arbeitsplätzen im formellen Sektor

1 Für die Welt insgesamt schätzt die ILO für 2020 einen Arbeitsstundenverlust von 8,8 Prozent. 
geprägt. Zwischen 2014 und 2019 wuchs die lateinamerikanische Wirtschaft im jährlichen Durchschnitt um lediglich 0,3 Prozent und die Zahl der Lohnarbeitsplätze jährlich nur um 0,7 Prozent. ${ }^{2}$ Wie in Lateinamerika üblich, äußerten sich diese Probleme bei der Generierung von Lohnarbeitsplätzen auf zweierlei Weise:

- Der informelle Sektor expandierte: Die Zahl der - mehrheitlich informellen - Selbständigen ${ }^{3}$ wuchs im jährlichen Durchschnitt um 2,4 Prozent, also mehr als dreimal so stark wie die - mehrheitlich formelle - Lohnarbeit.

- Die offene Arbeitslosigkeit stieg von 6,3 Prozent (2013) auf 8,0 Prozent (2019) an.

Vor diesem Hintergrund trafen die Pandemie und die ab März 2020 ergriffenen Maßnahmen zur Beschränkung der Ausbreitung des Virus SARSCoV-2 - vor allem die Stilllegung der wirtschaftlichen Aktivitäten, die nicht als essentiell wichtig klassifiziert wurden, sowie Mobilitätsbeschränkungen - die lateinamerikanischen Arbeitsmärkte mit äußerster Härte. Im zweiten Quartal 2020 fiel die Erwerbstätigenquote im Vergleich zum gleichen Vorjahreszeitraum von 57,4 Prozent auf 47,4 Prozent, was einem (zum Teil vorübergehenden) Verlust von etwa 47 Millionen Arbeitsplätzen entsprach. ${ }^{4}$

Anders als in Krisen wirtschaftlichen Ursprungs führten besagte Maßnahmen gleichzeitig zu einem massiven Rückzug von Personen vom Arbeitsmarkt, die ihr Arbeitseinkommen verloren. Die Erwerbsquote fiel zwischen dem 2. Quartal 2019 und dem gleichen Zeitraum 2020 von

2 Die lateinamerikanische Erwerbsbevölkerung (die Summe von Erwerbstätigen und Arbeitslosen) weist ein jährliches Trendwachstum von 1,1 Prozent auf, was als Mindesterfordernis der Schaffung von produktiven Arbeitsplätzen verstanden werden kann.

3 Wenn hier und in der Folge von Selbständigen die Rede ist, sind solche ohne Lohnarbeiter gemeint (trabajadores por cuenta propia).

4 Die Erwerbstätigenquote bezeichnet den Anteil, den die Erwerbstätigen an der Bevölkerung im arbeitsfähigen Alter ausmachen. Die hier und in der Folge genannten Arbeitsmarktdaten für 2020 und Anfang 2021 repräsentieren - bei den unterschiedlichen Variabeln - zwischen neun und 14 Länder, die monatlich oder quartalsweise Daten erheben. Die üblichen Methoden zur Datenerhebung mussten zeitweise modifiziert und auf telefonische Befragungen beschränkt werden, was die Vergleichbarkeit zu früheren Zahlen etwas beeinträchtigt. Einige Umfragen fielen ganz aus. 
62,7 Prozent auf 53,2 Prozent. ${ }^{5}$ Da nur erwerbslose Personen, die aktiv einen Arbeitsplatz suchen, als offen arbeitslos gelten, milderte dies den Anstieg der statistisch erfassten Arbeitslosigkeitsrate. Die offene Arbeitslosigkeit wuchs daher im gleichen Zeitraum ,lediglich'von 8,5 auf 11,0 Prozent. Dies spiegelte das Ausmaß der Arbeitsplatzvernichtung jedoch nicht annähernd wider. ${ }^{6}$

Während in Lateinamerika Krisen wirtschaftlichen Ursprungs zu einer Expansion des informellen Sektors führen, war dies im Kontext der Pandemie nicht der Fall. Vielmehr trafen die Beschränkungen von Aktivitäten, die in einem großen Ausmaß persönliche Kontakte erfordern, besonders den informellen Sektor.

Abb. 1: Lateinamerika (10 Länder): Veränderung der Zabl der Erwerbstätigen, nach Branchen, 2. Quartal 2020 / 2. Quartal 2019 (in Prozentzahlen)

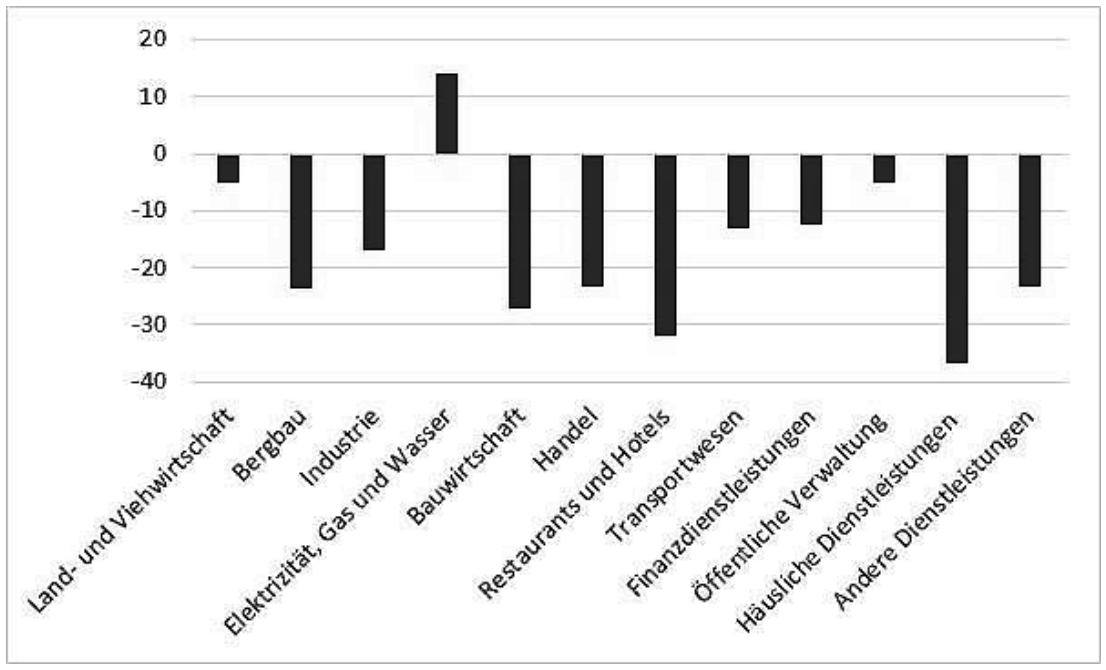

Wie aus Abb. 1 ersichtlich, war der Rückgang der Beschäftigung im 2. Quartal 2020 am größten bei den häuslichen Dienstleistungen, im Hotelund Gaststättengewerbe, in der Bauwirtschaft und im Handel. In vielen

5 Die Erwerbsquote bezeichnet den Anteil, den die auf dem Arbeitsmarkt befindlichen Personen (Erwerbsbevölkerung=Erwerbstätige plus Arbeitslose) an der Bevölkerung im arbeitsfähigen Alter ausmachen.

6 Wenn die Arbeitslosenquote im 2. Quartal 2020 mit der Erwerbsquote des 2. Quartals 2019 berechnet wird, beläuft sie sich auf 24,5 Prozent. 
dieser Branchen ist der Anteil des informellen Sektors überdurchschnittlich hoch und anders als formelle Unternehmen können informelle meist nicht auf Alternativen wie Telearbeit zurückgreifen. Folglich war der Beschäftigungsrückgang in den Sektoren am größten, die eine höhere Informalität aufweisen, speziell unter Lohnabhängigen mit Privathaushalten als Arbeitgeber, sowie Selbständigen und - zumeist damit verknüpft unbezahlt mitarbeitenden Familienangehörigen (Abb. 2).

\section{Abb. 2: Lateinamerika (11 Länder): Veränderung der Zahl der Erwerbstätigen, nach Beschäftigungskategorie, 2. Quartal 2020 / 2. Quartal 2019 (in Prozentzablen)}

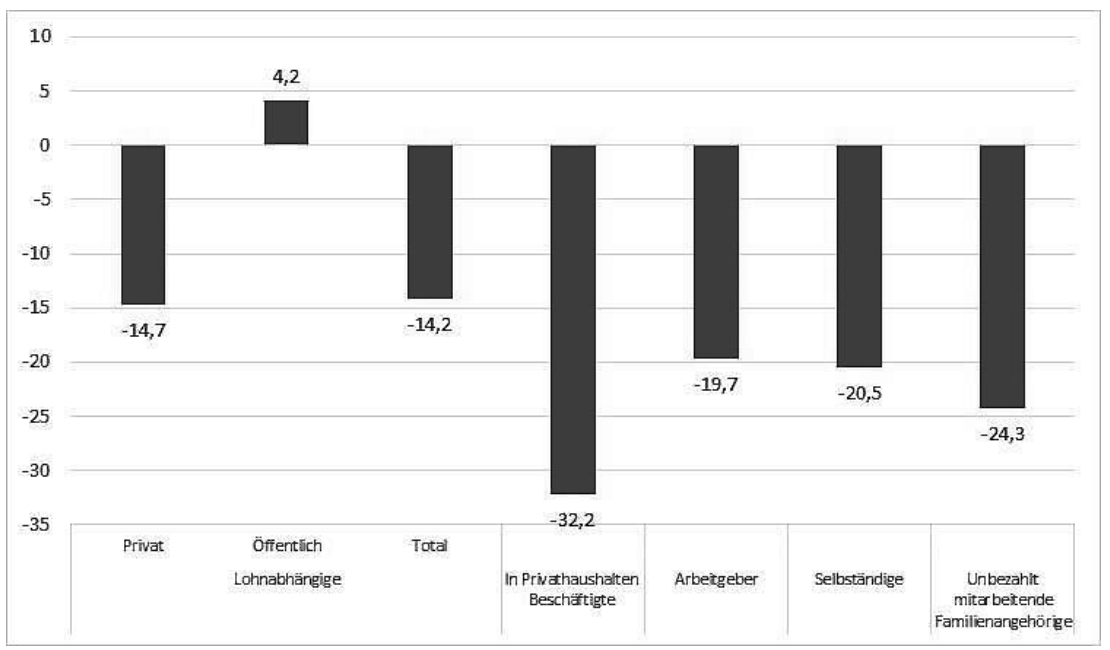

Quelle: Eigene Berechnung auf der Grundlage von CEPAL / OIT (2020).

Da die informelle Beschäftigung stärker fiel als die formelle, sank - entgegen der in Krisen üblichen Tendenz - die Informalitätsrate im 2. Quartal 2020, z.B. in Brasilien, Chile und Peru um 4,0, 5,3, bzw. 8,1 Prozentpunkte.

Der stärkere Fall der Beschäftigung im informellen Sektor trägt zur Erklärung bei, warum das regionale Bruttosozialprodukt viel weniger sank als die Zahl der Arbeitsstunden, da der Beitrag der Arbeitsplätze zum Wirtschaftswachstum in diesem Sektor deutlich geringer ist als im formellen.

Frauen waren von der Eliminierung von Arbeitsplätzen im 2. Quartal 2020 stärker betroffen als Männer. Dies erklärt sich vor allem dadurch, dass sie in einigen Branchen mit dem größten Verlust von Arbeitsplätzen 
einen überdurchschnittlich hohen Anteil an der Beschäftigung haben, insbesondere in den Bereichen häusliche Dienstleistungen, Handel und Hotel- und Gaststättengewerbe. So sank die Zahl der beschäftigten Frauen im Vorjahresvergleich um 18,1 Prozent, während die der Männer um 15,1 Prozent abnahm.

Wie aus Abb. 3 hervorgeht, konzentrierte sich der - im Vergleich zu den Männern - stärkere Rückgang der Frauenerwerbstätigkeit auf Beschäftigungskategorien, die generell höhere Informalitätsraten aufweisen: Selbständige, in Privathaushalten Beschäftigte und unbezahlte Familienmitarbeiterinnen. Vom starken Verlust informeller Einkommensmöglichkeiten waren folglich vorrangig Frauen betroffen.

Abb. 3: Lateinamerika (9 Länder): Veränderung der Zabl der Erwerbstätigen, nach Beschäftigungskategorie und Geschlecht, 2. Quartal 2020 / 2. Quartal 2019 (in Prozentzablen)

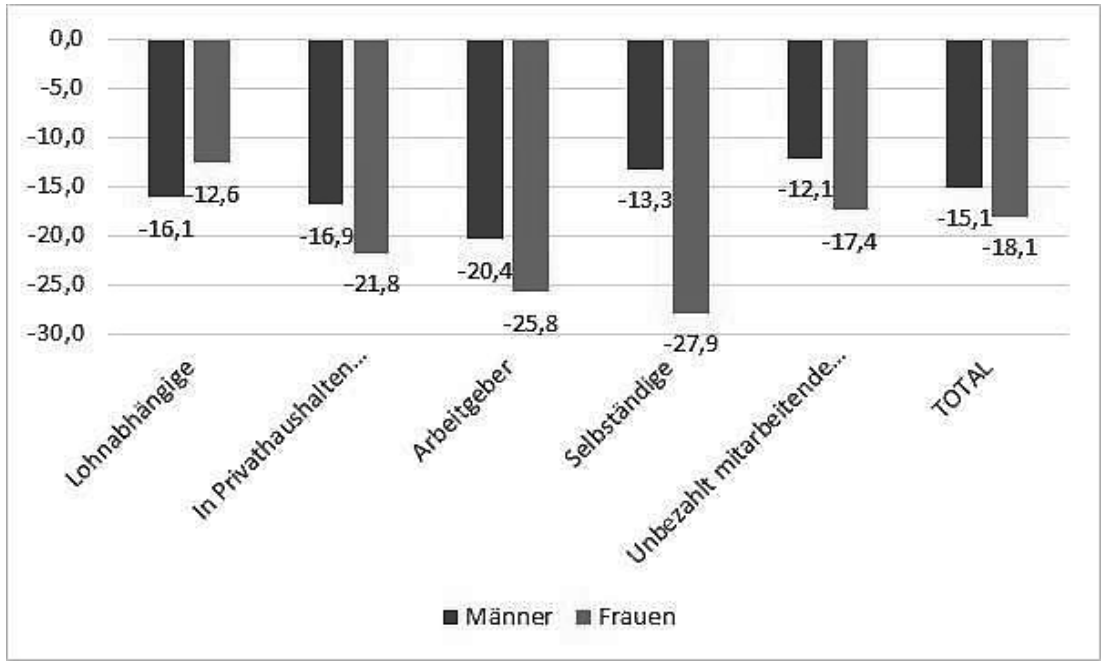

Quelle: Eigene Berechnung auf der Grundlage von offiziellen nationalen Zahlen.

Andererseits nahm die Zahl der Lohnarbeiterinnen weniger ab als die ihrer männlichen Pendants, was unter anderem an ihrem relativ hohen Anteil an der Beschäftigung in im Kontext der Pandemie als essenziell identifizierten Aktivitäten zurückzuführen ist (öffentliche Verwaltung, Gesundheits- und Bildungswesen).

Noch stärker als bei den Beschäftigten war der Unterschied zwischen Frauen und Männern im Fall der Erwerbsbevölkerung (-15,4 Prozent 
Frauen, -11,8 Prozent Männer), da sich ein größerer Anteil von Frauen vom Arbeitsmarkt zurückziehen musste, um sich im Zuge der Schließung von Kindergärten und Schulen der Betreuung ihrer Kinder zu widmen. Als Konsequenz dieser Verschiebungen stieg die Arbeitslosenrate der Männer (von 7,4 Prozent im 2. Quartal 2019 auf 10,1 Prozent im gleichen Zeitraum 2020) deutlicher als die der Frauen (von 10,0 Prozent auf 11,9 Prozent), ohne dass dies bedeuten würde, dass erstere stärker von den Auswirkungen der Pandemie auf dem Arbeitsmarkt betroffen wären vielmehr unterstreicht es die begrenzte Aussagekraft der Arbeitslosenrate im Kontext dieser speziellen Krise.

Dass die Beschäftigung im formellen Sektor weniger stark zurückging als im informellen, lag zum einen daran, dass Branchen mit relativ hohem Formalisierungsgrad weniger stark von den Maßnahmen zur Eindämmung der Pandemie betroffen waren, da einige von ihnen als essentiell wichtig galten. Zum anderen konnten formelle Unternehmen eine Reihe von Instrumenten nutzen, die ihren Weiterbetrieb ermöglichten und den Verlust von Arbeitsplätzen begrenzten. In der Tat griff ein beträchtlicher Prozentsatz der (formellen) Unternehmen - neben der Entlassung von Arbeitnehmern - auf Maßnahmen wie die Reduzierung der Zahl der Arbeitsstunden, vorzeitigen Urlaub und Telearbeit zurück (CEPAL/OIT 2020). ${ }^{7}$ In einigen Fällen wurden Strategien zur Beibehaltung von Arbeitsverhältnissen auch bei Produktionsstillstand durch Zahlungen aus der (nur in wenigen Ländern vorhandenen) Arbeitslosenversicherung oder durch öffentliche Transfers unterstützt (OIT 2020, BID 2021). Informationen über das Ausmaß der verschiedenen Maßnahmen liegen nur begrenzt vor, aber Daten einzelner Länder können einen Eindruck über ihre Relevanz verschaffen.

Die Kategorie der „abwesend Beschäftigten“ umfasst Personen, die in einem Arbeitsverhältnis stehen oder selbständig sind, in der Referenzwoche aber nicht gearbeitet haben, üblicherweise vor allem wegen Urlaub oder Krankheit. In der Pandemie stieg der Anteil der Abwesenden unter den Beschäftigten deutlich an, weil sie vorzeitigen Urlaub nahmen oder vom Staat oder einer Arbeitslosenversicherung Lohnersatz bekamen. In diesen Fällen blieb das Arbeitsverhältnis bestehen und die entsprechenden Arbeitnehmer galten weiterhin als beschäftigt. Der Anteil der Abwesenden unter den Beschäftigen stieg z.B. in Mexiko von 1,7 Prozent im März auf 21,9 Prozent im April 2020. Auch in Uruguay (23,7 Prozent im April),

7 CEPAL / OIT (2020) zeigt diesbezügliche Ergebnisse von Unternehmensbefragungen in verschiedenen Ländern der Region. 
Brasilien (18,6 Prozent im Mai), Argentinien und Chile (21,1 Prozent, bzw. 18,1 Prozent im 2. Quartal 2020) wurde dieses Instrument intensiv genutzt.

Gleichzeitig sank die Zahl der durchschnittlichen Arbeitsstunden unter den Beschäftigten, die weiterhin ihre Arbeit verrichten konnten. So erklärten etwa in Brasilien im Mai 2020 27,9 Prozent der „anwesend Beschäftigten", dass sie weniger als üblich arbeiteten. In Mexiko stieg der Anteil derjenigen, die weniger als 34 Wochenstunden arbeiten, von 23,1 Prozent im März auf 37,7 Prozent im April 2020.

Der hohe Anteil „abwesend Beschäftigter" und die geringere Zahl der Arbeitsstunden unter den „anwesend Beschäftigten“ unterstreicht, dass die Arbeitsmärkte von der Pandemie deutlich stärker getroffen wurden, als es der Fall der Beschäftigungsquote um 10 Prozentpunkte insinuiert. Dies führte dazu, dass die ILO (2021) den Verlust an Arbeitsstunden im 2. Quartal 2020 auf 32,8 Prozent schätzte. Entsprechend stark waren die Einkommensverluste selbst unter den Personen, die ihre Arbeit nicht verloren. Zum Beispiel berichteten im 3-Monats-Zeitraum Mai-Juli 2020 33,8 Prozent der Beschäftigten in Chile, dass sie pandemiebedingte Einkommensverluste hinnehmen mussten. Bei zwei Dritteln von ihnen beliefen sich diese auf die Hälfte oder mehr ihrer vorigen Arbeitseinkommen.

Die Möglichkeit zur Telearbeit verhinderte, dass der Rückgang des Arbeitsvolumens noch größer war. Zur Förderung dieser Arbeitsmodalität wurden 2020 und 2021 in mehreren Ländern der Region (Argentinien, Bolivien, Chile, El Salvador, Panama, Paraguay, Mexiko, Peru) gesetzliche Bestimmungen zu ihrer Regulierung erlassen. So stieg im Zuge der Maßnahmen zur Eindämmung der Pandemie der Anteil der („,anwesend“) Beschäftigten mit Telearbeit zum Beispiel in Brasilien auf 13,3 Prozent (Mai), in Costa Rica auf 15,3 Prozent (3. Quartal) und in Uruguay auf 19,3 Prozent (April). In den wenigen Ländern, die diesbezüglich über Information verfügen, war der Prozentsatz telearbeitender Frauen größer als der der Männer. Zum Beispiel belief er sich in Brasilien im Mai 2020 auf 17,9 Prozent bei den Frauen und auf 10,3 Prozent bei den Männern.

Schließlich wurde der Rückgang der Zahl der Erwerbstätigen durch den Anstieg der Nachfrage nach über digitale Plattformenvermittelten Dienstleistungen abgeschwächt, die aufgrund der Beschränkungen im Gastättengewerbe und im traditionellen Handel vor allem im elektronischen Handel und im Lieferservice anstieg (CEPAL 2020b). ${ }^{8}$ Aufgrund von Ar-

8 Zwischen dem 1. und dem 2. Quartal 2020 nahm der elektronische Handel in Lateinamerika um 157 Prozent zu (CEPAL 2020b). 
beitsplatzverlusten in anderen Bereichen suchten während der Pandemie viele Personen Einkommensalternativen in dieser Modalität (Alba et al. 2021).

Wie auch für OECD-Länder, wurde speziell für Lateinamerika projiziert, dass die Pandemie und die Maßnahmen zu ihrer Bekämpfung Niedrigeinkommenshaushalte stärker treffen würden als wohlhabendere Haushalte. Dies liegt an den entsprechenden Lebensbedingungen (beengte Wohnverhältnisse, von den Lebensumständen erzwungene Ignorierung von Quarantänemaßnahmen) und an den Verwerfungen auf dem Arbeitsmarkt (Restriktionen bezüglich Tätigkeiten, die persönliche Kontakte erfordern; Unmöglichkeit von Telearbeit) (Weller 2020; Delaporte et al. 2021).

Die tatsächliche Entwicklung des Arbeitsmarktes in der Pandemie bestätigte diese Annahme. Wie schon erwähnt, verloren in der Hochzeit der ersten Welle der Pandemie vor allem - großenteils zu Haushalten mit niedrigem pro-Kopf-Einkommen gehörende - informell Beschäftigte ihre Einkommensquellen. Ein weiterer Indikator für diese, die starke Ungleichheit auf den lateinamerikanischen Arbeitsmärkten verschärfende, Entwicklung ist die Beschäftigungsverlustrate für unterschiedliche Bildungsgruppen.

Abb. 4 zeigt exemplarisch den Anteil der Beschäftigungsgruppen mit dem niedrigsten (bis Grundschulabschluss) und dem höchsten (Universitätsstudien) Bildungsniveau im 1. und 2. Quartal 2020 in fünf lateinamerikanischen Ländern. In vier von ihnen nahm der Anteil der niedrigsten Bildungsgruppe deutlich ab, während der der höchsten anstieg. ${ }^{9}$

Die höheren Verluste von Arbeitseinkommen von Beschäftigten mit niedriger formeller Bildung lag daran, dass sie zum einen hauptsächlich im informellen Sektor arbeiten, zum anderen an geringeren Zugang zu Telearbeit. ${ }^{10}$

9 Zahlen für Lima weisen dasselbe Muster der anderen Länder auf. Gleichzeitig nahm in Peru die Beschäftigung in der Landwirtschaft stark zu, was auf StadtLand-Migrationsbewegungen und neue Arbeitsverhältnisse von Personen mit relativ niedrigem Bildungsgrad hindeutet.

10 Im Mai 2020 belief sich in Brasilien der Anteil der Beschäftigten mit Telearbeit auf 0,6 Prozent bei Personen ohne Grundschulabschluss, 1,7 Prozent bei Personen mit Grundschulabschluss, 7,9 Prozent bei Personen mit Sekundärschulabschluss und 38,3 Prozent bei Personen mit Universitätsabschluss. 
Abb. 4: Lateinamerika (ausgewählte Länder): Anteil von Beschäftigten mit Bildung bis Grundschulabschluss und mit Universitätststudien an der Gesamtbeschäftigung, 1. und 2. Quartal 2020 (in Prozentzablen)

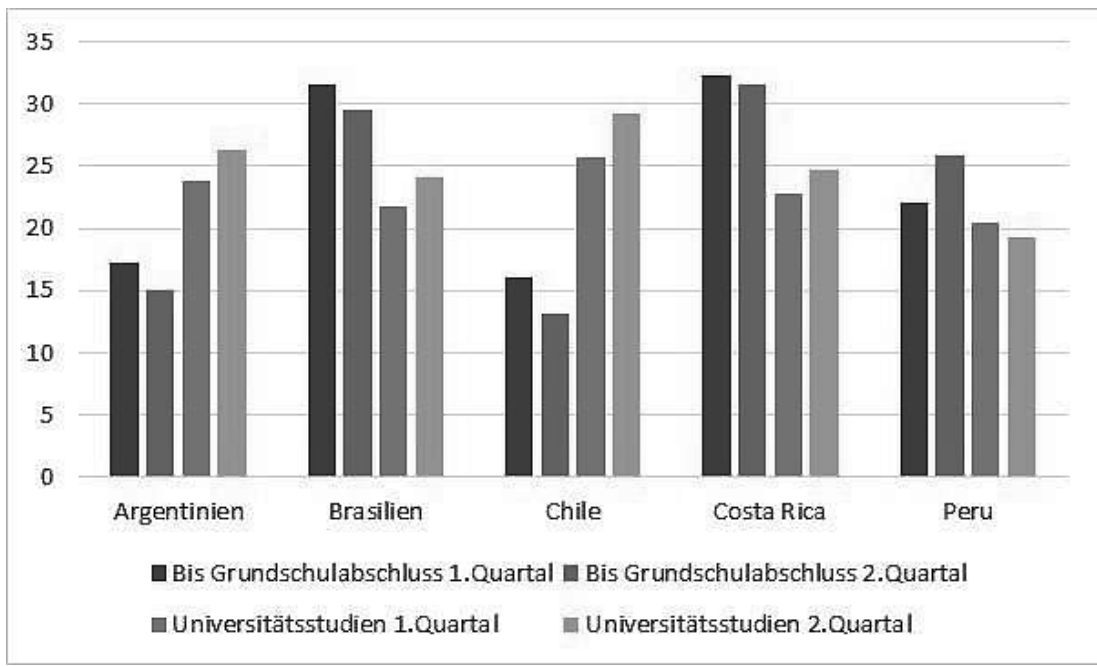

Quelle: CEPAL (2020a).

Eine weitere Beschäftigtengruppe mit überdurchschnittlich starken Arbeitsplatzverlusten waren junge Leute. Der Anteil der jüngsten Beschäftigtengruppe (bis 24 Jahre) nahm zwischen dem 1. und dem 2. Quartal 2020 in 11 Ländern der Region, über die diesbezügliche Informationen zugänglich sind, im Durchschnitt um 2,1 Prozentpunkte ab. Die Zahlen schwanken zwischen einem Rückgang von 0,7 Punkten in Paraguay und einem Höchstwert von 3,8 Punkten in Bolivien. In diesem Fall spielte die Telearbeit eine geringere Rolle. ${ }^{11}$ Allerdings nahm in der Pandemie die Zahl der Neueinstellungen drastisch ab, was junge Menschen (viele von ihnen neu auf dem Arbeitsmarkt) besonders hart zu treffen pflegt.

Schließlich waren Migranten besonders von den Auswirkungen der Pandemie auf den Arbeitsmärkten betroffen, vor allem in den häuslichen Dienstleistungen, im Handel und im Hotel- und Gaststättengewerbe (Carella 2021). Die Lage von Migranten verschlechterte sich vor allem, wenn

11 In Brasilien lag im Mai 2020 der Anteil der jungen Beschäftigten mit Telearbeit wenig unter dem der Beschäftigten insgesamt. 
sie aufgrund eines ungeregelten Migrationsstatus keinen Zugang zu öffentlichen Transferleistungen hatten.

Während zum Beispiel in den USA und europäischen Ländern eine ähnliche Verschärfung der Arbeitsmarktungleichheit im Kontext der Pandemie durch sozialpolitische Maßnahmen weitgehend ausgeglichen wurde, wurde diese in Lateinamerika nur punktuell durch Transferleistungen gemildert (ILO 2021: 15f.).

\section{Graduelle Erholung bei fortbestehenden Defiziten}

Nach dem Ende der ersten Pandemiewelle wurden in allen lateinamerikanischen Ländern die Restriktionen hinsichtlich wirtschaftlicher Aktivitäten und persönlicher Mobilität gelockert. Allerdings wurden sie später in Reaktion auf eine zweite und eine dritte Welle zuweilen wieder verschärft, so dass sich die Arbeitsmärkte nur graduell und mit Rückschlägen verbunden erholten. Speziell informell Beschäftigte nahmen zur Überlebenssicherung oft ihre Arbeit auch da wieder auf, wo es die offiziellen Richtlinien noch nicht wieder zuließen.

Wie aus Abb. 5 ersichtlich, erholte sich die Erwerbstätigenquote ab dem 3. Quartal 2020 langsam. Nachdem sie im 2. Quartal 2020, wie erwähnt, um 10 Prozentpunkte unter dem Wert des gleichen Vorjahreszeitraums gelegen hatte, betrug diese Lücke im 4. Quartal 2020 noch 4,7 und im 1. Quartal 2021 3,3 Prozentpunkte. ${ }^{12}$

Gleichzeitig kehrte angesichts graduell wieder erweiterter Arbeitsgelegenheiten ein wachsender Anteil der Personen auf den Arbeitsmarkt zurück, die ihn während der ersten Welle verlassen hatten. Dies manifestierte sich in einer schrumpfenden Lücke der Erwerbsquote im Jahresvergleich. Daher führte der Anstieg der Beschäftigung nicht unmittelbar zu einem Rückgang der Arbeitslosenquote. Im Jahresvergleich stieg die Arbeitslosigkeit im 3. Quartal 2020 (3,0 Prozentpunkte höher als im selben Zeitraum 2019) gegenüber dem 2. Quartal sogar noch an. Erst im 1. Quartal 2021 war eine nennenswerte Verbesserung zu registrieren, auch wenn die Arbeitslosenquote mit 10,8 Prozent immer noch 1,9 Prozentpunkte über dem Vorjahreswert lag.

12 Hinsichtlich des Jahresvergleichs im 1. Quartals 2021 ist zu berücksichtigen, dass die Maßnahmen zur Eindämmung der Pandemie i.a. Mitte März 2020 ergriffen wurden und die Zahlen des 1. Quartals 2020 bereits erste Verwerfungen auf dem Arbeitsmarkt widerspiegelten, wie in Abb. 5 zu sehen ist. 
Abb. 5: Lateinamerika und Karibik (14 Länder): Erwerbstätigen-, Erwerbs- und Arbeitslosenquote, Veränderung gegenüber dem Vorjahr, 4. Quartal 2019 bis 1. Quartal 2021 (in Prozentpunkten)

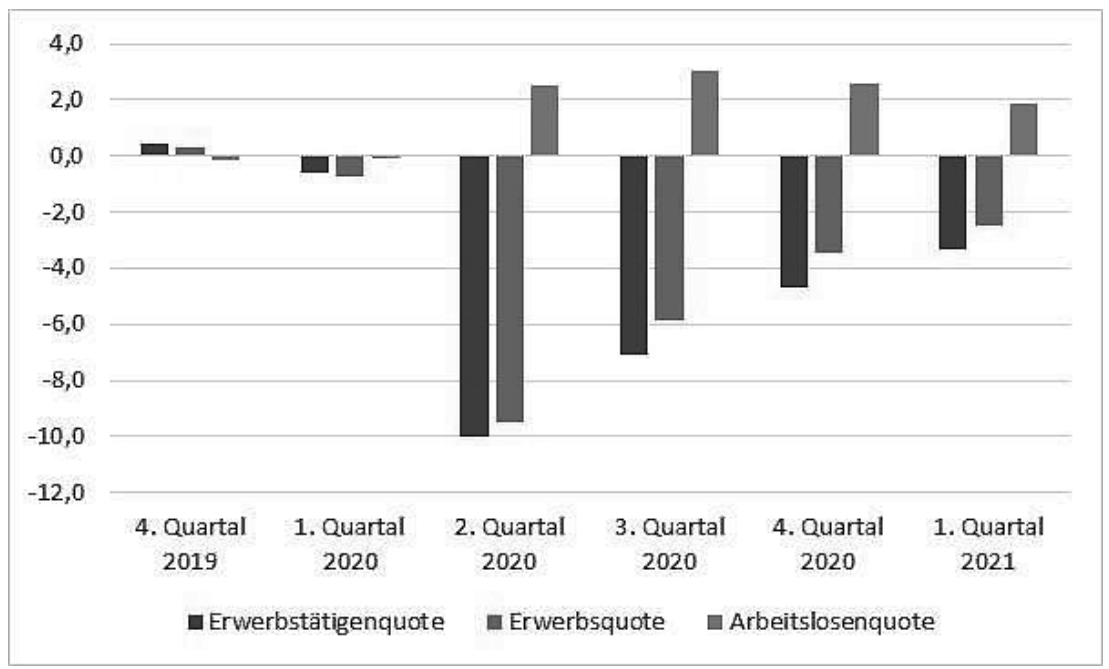

Quelle: Eigene Berechnung auf der Grundlage von offiziellen nationalen Zahlen.

Die Abb. 6 zeigt große Unterschiede sowohl des Falls der Erwerbstätigenquote im 2. Quartal 2020 als auch hinsichtlich der darauffolgenden Erholung. In Peru fiel diese Quote im 2. Quartal 2020 am stärksten; auch in Argentinien, Chile, Costa Rica, Ecuador, Kolumbien und Mexico sank sie um 10 oder mehr Prozentpunkte. Die geringsten Arbeitsplatzverluste verzeichneten Nicaragua, Paraguay und Uruguay.

Trotz ihres graduellen Wiederanstiegs ab dem 3. Quartal 2020 lag diese Quote in Brasilien, Chile und Costa Rica im 1. Quartal 2021 noch um mehr als 5 Prozentpunkte unter dem Vorjahreswert, während sie in Argentinien, Bolivien, Ecuador, Nicaragua, Paraguay und Uruguay nahe oder sogar oberhalb dieses Vergleichswerts lag. 
Abb. 6: Lateinamerika (ausgewählte Länder): Veränderung der Erwerbstätigenquote gegenüber dem Vorjahr, 2. Quartal 2020 und 1. Quartal 2021 (in Prozentpunkten)

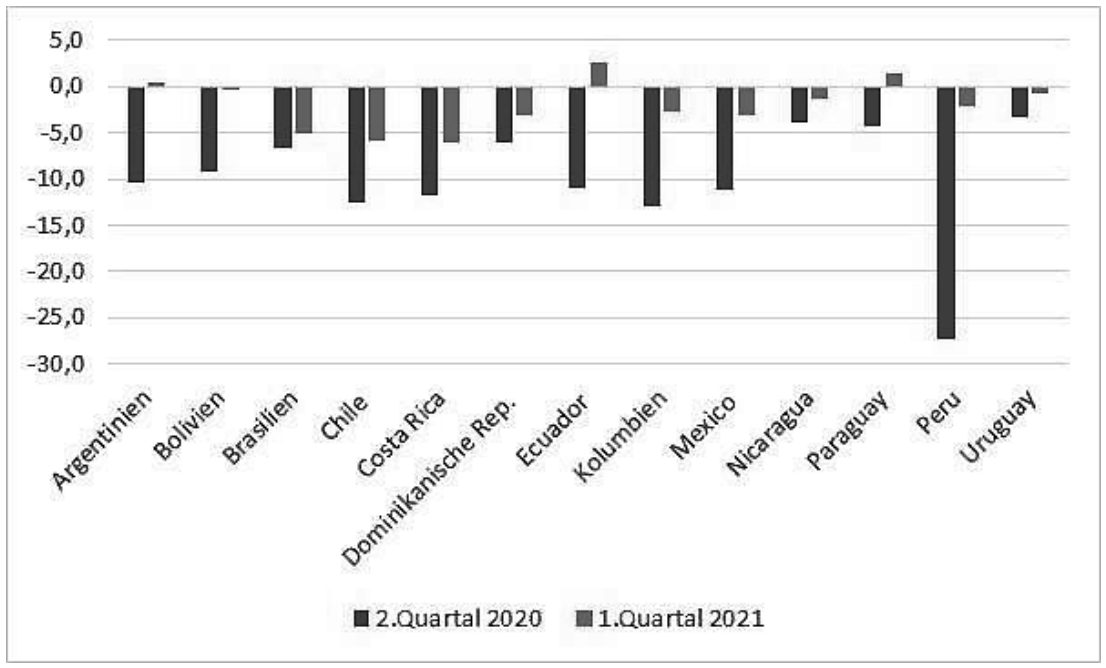

Quelle: Eigene Berechnung auf der Grundlage von offiziellen nationalen Zahlen.

Die graduelle Rückkehr auf den Arbeitsmarkt konzentrierte sich zunächst auf die Männer. Im 3. Quartal 2020 betrug der Arbeitsplatzverlust bei Männern gegenüber dem gleichen Vorjahresquartal 9,1 Prozent (nach 15,4 Prozent im 2. Quartal), während er sich bei den Frauen noch auf 14,3 Prozent belief (gegenüber 18,1 Prozent im 2. Quartal). Bis zum 1. Quartal 2021 reduzierte sich diese Differenz zwischen Männern und Frauen, aber der Arbeitsplatzverlust bei Frauen war im Vergleich zum gleichen Quartal des Vorjahrs weiterhin größer als bei Männern (-6,9 Prozent und -3,6 Prozent). Dies spiegelt zumindest zum Teil den stärkeren Rückgang der weiblichen Erwerbsbevölkerung wider (-3,9 Prozent im Jahresvergleich im 1. Quartal 2021 versus -2,0 Prozent bei Männern), die im Wesentlichen auf die ungleiche Verteilung der Reproduktionsarbeit im Haushalt zurückzuführen ist.

Wie erwähnt, trafen die Maßnahmen zur Eindämmung der Pandemie im 2. Quartal die in Privathaushalten Beschäftigten sowie die hauptsächlich informellen Beschäftigungskategorien der Selbständigen und der unbezahlt mitarbeitenden Familienmitglieder am stärksten. Das aus Subsistenzerfordernissen notwendige Ignorieren der Restriktionen und ihre graduelle Aufhebung führten dazu, dass bereits ab dem 3. Quartal bei den 
beiden letztgenannten Kategorien der Rückgang im Vorjahresvergleich geringer war als bei den Lohnarbeiter"innen. Im 1. Quartal 2021 lag die Zahl der Selbständigen nur noch um 0,7 Prozent unter dem Vorjahreswert, während die der unbezahlt mitarbeitenden Familienmitglieder diesen Vergleichswert sogar um 1,8 Prozent übertraf (Abb. 7).

Abb. 7: Lateinamerika (10 Länder): Veränderung der Zabl der Erwerbstätigen, nach Beschäftigungskategorie, 2. Quartal 2020 / 2. Quartal 2019 und 1. Quartal 2021 / 1. Quartal 2020 (in Prozentzablen)

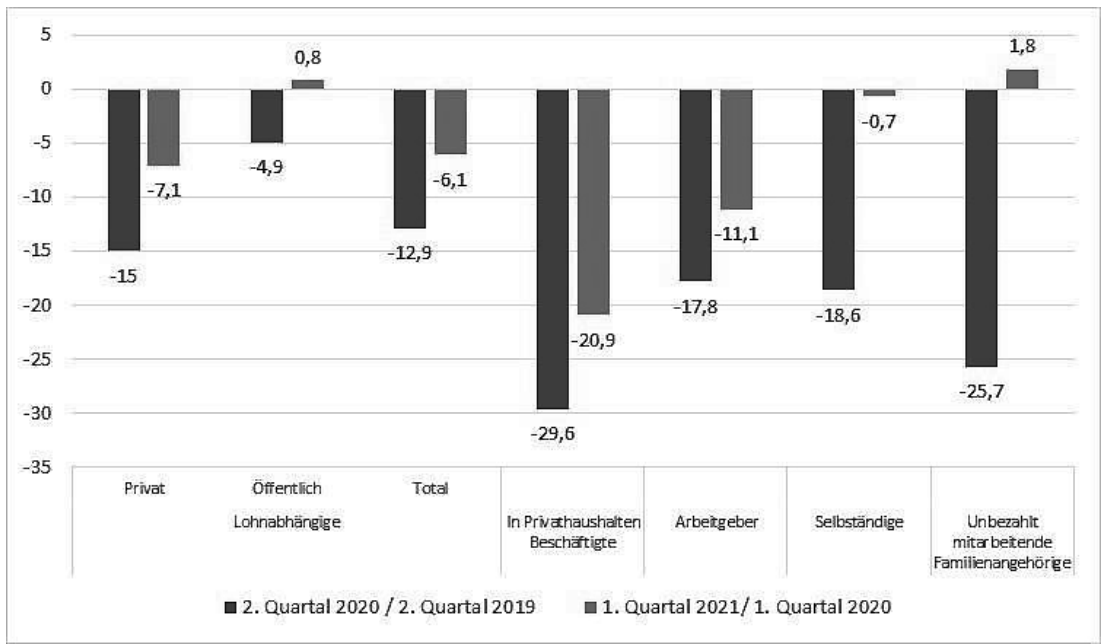

Quelle: Eigene Berechnung auf der Grundlage von offiziellen nationalen Zahlen.

Den größten Beschäftigungsrückgang im Vorjahresvergleich verzeichneten auch im 1. Quartal 2021 die in Privathaushalten Beschäftigten (-20,9 Prozent), was zum anhaltend größeren Arbeitsplatzverlust im Jahresvergleich bei Frauen beigetragen hat. Auch die Beschäftigungslücke bei Arbeitgebern nahm nur geringfügig ab (-11,1 Prozent). Dies verweist auf die Schwierigkeiten vor allem von Kleinunternehmen, ihren Betrieb wieder aufzunehmen, sowie auf eine geringe Zahl von Geschäftsneugründungen. Die Zahl der bei Privatunternehmen oder im öffentlichen Dienst beschäftigten Lohnabhängigen lag zu Beginn von 2021 um 7,1 Prozent unter dem Vorjahresniveau.

Die stärkere Zunahme der Beschäftigung in vorwiegend informellen Beschäftigungskategorien führte zu einem Wiederanstieg der Informalitätsquote ab dem 3. Quartal 2020. Im 1. Quartal 2021 lag diese im Durch- 
schnitt von neun Ländern auf der gleichen Höhe wie im Vorjahreszeitraum. In der Dominikanischen Republik, Kolumbien, Paraguay und Peru übertraf sie sogar den entsprechenden Vergleichswert.

Von den ursprünglich am stärksten von den Maßnahmen zur Eindämmung der Pandemie betroffenen Branchen wiesen im 1. Quartal 2021 das Hotel- und Gaststättengewerbe (-19,5 Prozent) und die häuslichen Dienstleistungen (-20,6 Prozent) noch den größten Rückgang der Beschäftigungszahlen im Vorjahresvergleich auf. Die Beschäftigung bei den sonstigen Dienstleistungen (Rückgang der Lücke zum Vorjahr auf -16,0 Prozent) und vor allem im Bergbau (-6,3 Prozent), der Bauwirtschaft (-2,6 Prozent) und im Handel (-7,5 Prozent) nahm vergleichsweise stärker zu. Branchen wie die Land- und Viehwirtschaft, die öffentliche Verwaltung, das Erziehungs- und Gesundheitswesen, die Elektrizitäts-, Gas- und Wasserversorgung erreichten bereits im 4. Quartal 2020 das Beschäftigungsniveau des gleichen Vorjahreszeitraums.

Der vergleichsweise geringe Wiederanstieg der Zahl der Lohnarbeiter*innen hatte zum Teil mit der Nutzung der Mechanismen zu tun, die während der ersten Welle der Pandemie ihren Fall abbremste. So kehrte zunächst ein beträchtlicher Teil der „abwesend Beschäftigten“ in den jeweiligen Arbeitsprozess zurück. Zum Beispiel sank der Anteil dieser Beschäftigtengruppe unter den Lohnabhängigen in Argentinien zwischen dem 2. und dem 4. Quartal 2020 von 21,2 Prozent auf 6,3 Prozent und in Mexiko zwischen April und Dezember 2020 von 27,0 Prozent auf 3,0 Prozent. Auch Brasilien, Chile und Uruguay verzeichneten deutliche Rückgänge der „abwesend Beschäftigten“. ${ }^{13}$

Gleichzeitig nahm auch die Telearbeit wieder ab. Zum Beispiel sank der Anteil der Beschäftigten mit Telearbeit in Uruguay von 19,3 Prozent im April 2020 auf 11,3 Prozent zum Jahresende, und in Chile nahm der Anteil der Beschäftigten, die zu Hause arbeiten, von 19,8 Prozent im 2. und 21,2 Prozent im 3. Quartal 2020 auf 13,9 Prozent im 1. Quartal 2021 ab. Es ist allerdings festzuhalten, dass der Anteil der Telearbeit weiterhin deutlich über dem vorpandemischen Niveau lag. Zum Beispiel arbeiteten im 1. Quartal 2020 in Argentinien nur 2,6 Prozent der Lohnabhängigen mit eigenem Arbeitsgerät, während es ein Jahr später, auch nach dem Rückgang gegenüber dem Maximum von 16,1 Prozent im 3. Quartal 2020, im 1. Quartal 2021 noch 11,5 Prozent waren. In Costa Rica belief sich der Anteil der Telearbeit im 1. Quartal von 2020 und 2021 auf 1,5 Prozent,

13 Zu Beginn des Jahres 2021 stieg in mehreren Ländern dieser Prozentsatz wieder an, allerdings hauptsächlich, weil es sich um die Haupturlaubszeit handelt. 
bzw. 10,2 Prozent. Diese anhaltend hohen Quoten liegen zum einen an Restriktionen, die zum Teil wieder verschärft wurden - in Chile und Uruguay nahm der Anteil der Telearbeit im 1. Quartal 2021 gegenüber dem 4. Quartal 2020 wieder zu -, zum Teil auch daran, dass Unternehmen und Beschäftigte Vorteile der Telearbeit feststellten, die pandemieunabhängig sind (CEPAL 2021).

\section{Arbeitsmarkttendenzen und Covid-19}

Die weitere Entwicklung der lateinamerikanischen Arbeitsmärkte hängt zum Teil von der Reaktivierung des Produktionsapparates und dementsprechend vom Wirtschaftswachstum ab. Wenngleich nach dem starken Rückgang des regionalen BIP in 2020 die Wirtschaft der Region im Jahr 2021 expandierte, hängt das Ausmaß dieser Reaktivierung unter anderem vom schwer vorauszusehenden weiteren Verlauf der Pandemie ab. Nicht nur traf viele lateinamerikanische Länder in den ersten Monaten 2021 ein neuer Anstieg von Ansteckungs- und Todesfällen, sondern die Ungewissheit bezüglich der Auswirkungen neuer Mutationen von SARS-CoV-2 erschwert Projektionen bezüglich des Wirtschaftswachstums für 2021 und die folgenden Jahre. ${ }^{14}$

Diese Ungewissheit droht auch das längerfristige Wachstum zu mindern, da sie Privatinvestitionen hemmt. Gleichzeitig ist der Spielraum für öffentliche Investitionen in vielen Ländern durch den Anstieg der Staatsverschuldung im Kontext der Pandemie eingeschränkt. All dies bremst die Schaffung neuer Arbeitsplätze im formellen Sektor, und es ist davon auszugehen, dass die Informalitätsrate der meisten Ländern in den nächsten Jahren steigen wird.

Außerdem wird sich die Erwerbsquote graduell erholen, vor allem, wenn in den Schulen wieder weitgehend Präsenzunterricht betrieben werden kann. Daher dürfte die begrenzte Schaffung von (formellen und informellen) Arbeitsplätzen kaum ausreichen, die Arbeitslosigkeit spürbar zu senken. Gleichzeitig ist ungewiss, ob - wie in den letzten Jahrzehnten die Lücke der Erwerbsquote zwischen Männern und Frauen sich wieder

14 Die CEPAL projiziert ein Wirtschaftswachtum von 5,9 Prozent für 2021 und 2,9 Prozent für 2022 (CEPAL 2021). 
reduziert, nachdem diese Tendenz während der Pandemie umgekehrt wurde. ${ }^{15}$

Außerdem beschleunigten sich im Kontext der Pandemie verschiedene Tendenzen, die im Zusammenhang mit digitalen Technologien stehen, speziell die Automatisierung, die Arbeit für digitale Plattformen und die Telearbeit (Weller 2020; McKinsey Global Institute 2021). Diese Tendenzen dürften die lateinamerikanischen Arbeitsmärkte in der Zukunft verstärkt prägen.

Aufgrund struktureller Eigenheiten der lateinamerikanischen Produktionsstrukturen und Arbeitsmärkte ist das Automatisierungspotential in der Region aber deutlich geringer als in entwickelten Ländern (Weller et al. 2019). Wenngleich keine genauen Daten vorliegen, weisen punktuelle Informationen allerdings darauf hin, dass die Pandemie die Automatisierungsprozesse auch in Lateinamerika beschleunigt. So erklärten zwischen 56 Prozent (in Argentinien) und 84 Prozent (in Mexiko) der für das World Economic Forum (2020) befragten Großunternehmen, dass sie als Reaktion auf die Pandemie die Automatisierung stärker vorantreiben.

Speziell der Onlinehandel expandierte während der Quarantänephasen. Auch wenn der Präsenzhandel danach wieder zugenommen hat, ist zu erwarten, dass ein beträchtlicher Anteil der während der Pandemie neuen Kunden des elektronischen Handels diesen nicht wieder vollständig aufgeben werden und der Onlinehandel tendenziell an Bedeutung gewinnt. Entsprechend entstehen neue Beschäftigungsmöglichkeiten, begleitet von einem Arbeitsplatzabbau im Präsenzhandel.

Wie erwähnt, nahm in diesem Zusammenhang vor allem die Beschäftigung über digitale Lieferservice-Plattformen während der Quarantänephasen deutlich zu. Auch die globale Nachfrage für digitale Arbeiten, die auch für Beschäftigungsmöglichkeiten lateinamerikanischer qualifizierter Dienstleister relevant ist, beschleunigte sich während der Pandemie wenn auch mit starken, von den verschiedenen Phasen der Pandemie beeinflussten, Schwankungen (CEPAL 2021). Auch in diesem Fall ist nicht zu erwarten, dass die entsprechenden Arbeitsmöglichkeiten auf das VorPandemieniveau zurückfallen. Gleichzeitig ging zwar die Nachfrage nach plattformvermittelten Präsenzdienstleistungen (v.a. Personentransport) zu-

15 Zwar argumentieren z.B. Alon et al. (2020), dass eine Quarantänesituation die häusliche Arbeitsteilung verändern könnte; allerdings modifizierte die Pandemie z.B. in Italien traditionelle Geschlechterrollen nicht einmal, wo Männer einen größeren Anteil der Hausarbeit übernahmen (Del Boca et al. 2021). Zukünftige Studien werden zeigen, welche Rolle die Pandemie diesbezüglich in Lateinamerika spielt. 
rück; aber dies ist vermutlich nicht dauerhaft, da häufig speziell die öffentlichen Transportmittel als Risikoumfeld potenzieller Ansteckung gewertet werden.

Gleichzeitig beschleunigte die Pandemie die Anwendung von Telearbeit. Wie auch in anderen Regionen stellt sich die Frage nach den zukünftigen Charakteristiken dieser Arbeitsmodalität, die sowohl für die Arbeitnehmer als auch für die Unternehmen potenzielle Vor- und Nachteile mit sich bringt (CEPAL 2021; Maurizio 2021).

Schließlich hat die Pandemie globale Wertschöpfungsketten getroffen, deren Expansion bereits vorher aus unterschiedlichen Gründen gebremst worden war. ${ }^{16}$ Es ist zu erwarten, dass die in diesem Zusammenhang ergriffenen Maßnahmen, darunter die Beschleunigung von Automatisierungsprozessen, fortgeführt werden (Valenzuela/Reinecke 2021).

\section{Schlussbemerkungen}

Die Covid-19 Pandemie hat die lateinamerikanischen Arbeitsmärkte in die tiefste Krise gestürzt, seit es systematische Datenerhebungen gibt. Anders als bei Krisen, die einen wirtschaftlichen Ursprung haben, hat in dieser Situation der informelle Sektor keine „Pufferwirkung“ entwickeln können. Vielmehr trafen ihn die Maßnahmen zur Eindämmung der Pandemie stärker als den formellen Sektor, da in ihm Aktivitäten überwiegen, die persönliche Präsenz erfordern und technologisch mögliche Alternativen wie Telearbeit aus verschiedenen Gründen meist nicht in Frage kommen.

Ein weiterer Unterschied zu Krisen wirtschaftlichen Ursprungs war der Rückzug eines beträchtlichen Teils der Erwerbsbevölkerung vom Arbeitsmarkt. Daher war der Anstieg der offenen Arbeitslosigkeit deutlich geringer, als der Rückgang der Zahl Erwerbstätiger impliziert hätte.

Der massive Verlust von Arbeitseinkommen, der im 2. Quartal 2020 seinen Höhepunkt hatte und in den folgenden Quartalen nur langsam rückgängig gemacht werden konnte, vertiefte bestehende Ungleichheiten auf dem Arbeitsmarkt, da neben den informell Beschäftigten Frauen, junge Menschen, Personen mit relativ niedrigem Bildungsniveau und Migranten besonders stark von ihm betroffen waren.

$\mathrm{Ab}$ dem 3. Quartal 2020 erholten sich die lateinamerikanischen Arbeitsmärkte langsam und nachdem die Erwerbstätigenquote im 2. Quartal 2020

16 Zum Beispiel musste die Handyproduktion in Brasilien aus Mangel an Zwischenprodukten zeitweise eingestellt werden (Valenzuela/Reinecke 2021). 
einen Fall von 10,0 Prozentpunkten gegenüber dem Vorjahresniveau erlitt, lag sie im 1. Quartal 2021 3,3 Prozentpunkte unter dem Niveau des 1. Quartals 2020, wobei sich der Anstieg des Beschäftigungsniveaus auf informelle Arbeitsverhältnisse konzentrierte.

Die Maßnahmen zur Eindämmung der Pandemie beschleunigten einige schon bestehende Tendenzen auf dem Arbeitsmarkt, vor allem Automatisierungsprozesse, Telearbeit und von digitalen Plattformen organisierte Arbeitsverhältnisse. Dies forciert zum Teil die Prekarisierung des Arbeitsmarktes. Die politischen Prioritäten in diesem Bereich müssen in diesem Zusammenhang die Förderung neuer formeller Arbeitsplätze, die effektive und inklusive produktive Nutzung neuer Technologien und die angemessene, partizipativ zu entwickelnde Regulierung der neu entstehenden Beschäftigungsverhältnisse sein.

\section{Literatur}

Alba Vega, Carlos; Bensusán, Graciela; Vega, Gustavo (2021): El trabajo del futuro con derechos laborales: diagnóstico y estrategia de política pública para el reconocimiento de derechos laborales de personas trabajadoras por plataformas digitales, Ciudad de México: El Colegio de México.

Alon, Titan M.; Doepke, Matthias; Olmstead-Rumsey, Jane; Tertilt, Michèle (2020): The Impact of COVID-19 on Gender Equality, NBER Working Paper Series, Working paper 26947.

BID (2021): Convivir con el Coronavirus. ¿Proteger el empleo o los ingresos de los trabajadores?, Washington, D.C.

Carella, Francesco; mit Frean, Silvi und Velasco, José Jacobo (2021): Migración laboral, movilidad, en el mundo del trabajo ante la pandemia de la COVID-19 en América Latina y el Caribe, Panorama Laboral en tiempos de la COVID-19, in: Nota Técnica, OIT, Lima.

CEPAL (2021): Estudio económico de América Latina y el Caribe 2021, Santiago de Chile: CEPAL

CEPAL (2020a): Balance preliminar de las economías de América Latina y el Caribe 2020, Santiago de Chile: CEPAL

CEPAL (2020b): Universalizar el acceso a las tecnologías digitales para enfrentar los efectos del COVID-19, Santiago de Chile: CEPAL.

CEPAL; OIT (2021): Trabajo decente para los trabajadores de plataformas en América Latina, in: Coyuntura Laboral en América Latina y el Caribe, 24. Santiago de Chile: Naciones Unidas/OIT.

CEPAL; OIT (2020): La dinámica laboral en una crisis de características inéditas: desafíos de política, in: Coyuntura Laboral en América Latina y el Caribe, 23. Santiago de Chile: Naciones Unidas/OIT. 
Del Boca, Daniela; Oggero, Noemi; Profeta, Paola; Rossi, Maria Cristina (2021): Did Covid-19 Affect the Division of Labor within the Household? Evidence from Two Waves of the Pandemic in Italy, in: IZA DP, 14453.

Delaporte, Isaure; Escobar, Julia; Peña, Werner (2021): The Distributional Consequences of Social Distancing on Poverty and Labour Income Inequality in Latin America and the Caribbean, GLO Discussion Paper, No. 682.

ILO (2021): ILO Monitor: COVID-19 and the world of work. Seventh edition, Updated estimates and analysis, Geneva: ILO.

Maurizio, Roxana (2021): Desafíos y oportunidades del teletrabajo en América Latina y el Caribe, Lima: OIT.

McKinsey Global Institute (2021): The Future of Work after COVID-19, [https://www .mckinsey.com/featured-insights/future-of-work/the-future-of-work-after-covid-1 9], letzter Aufruf 30.12.2012.

OIT (2020): Panorama Laboral 2020, Lima: Organización Internaitonal del Trabajo.

Valenzuela, María Elena; Reinecke, Gerhard (2021): Impacto de la COVID-19 en cadenas mundiales de suministro en América Latina: Argentina, Brasil, Chile, Paraguay y Uruguay, in: Nota técnica, OIT, Lima.

Weller, Jürgen (2020): La pandemia del COVID-19 y su efecto en las tendencias de los mercados laborales, Documentos de Proyectos, Santiago de Chile: CEPAL.

Weller, Jürgen; Gontero, Sonia; Campbell, Susanna (2019): Cambio tecnológico y empleo: una perspectiva latinoamericana. Riesgos de la sustitución tecnológica del trabajo humano y desafíos de la generación de nuevos puestos de trabajo, in: serie Macroeconomía del desarrollo, 201, Santiago de Chile: CEPAL.

World Economic Forum (2020): The Future of Jobs Report 2020, Geneva. 
